\title{
Peripheral Chemoreceptors and Sudden Infant Death Syndrome: A Wide Open Problem
}

\author{
Luigi Matturri*,\#, Giulia Ottaviani" ${ }^{\#}$ Anna M. Lavezzi ${ }^{\#}$ and Simone G. Ramos ${ }^{\S}$ \\ \# "Lino" Rossi Research Center for the study and prevention of unexpected perinatal death (of term fetus - stillbirth-and \\ neonatal) and sudden infant death syndrome (SIDS), Institute of Pathology, University of Milan, Italy, ${ }^{\S}$ Department of \\ Pathology, Faculty of Medicine of Ribeirão Preto, University of São Paulo, Brazil.
}

\begin{abstract}
This review will focus on the basic mechanisms of the peripheral chemoreceptors elicited by studies on mature chemoreceptors and on the alterations observed in these structures related to sudden infant death. The integrated response of the peripheral chemoreceptors consists of the transduction of alterations in the chemical milieu, regulating the temperature of their environment to alterations in cardiovascular and respiratory performance. Over the years, much has been written about the relative importance of the carotid and aortic bodies in the peripheral chemoreceptor response, and it is therefore worthwhile to consider the current state-of-the-art on that issue. Several lines of evidence have suggested that abnormalities in chemoreception may play a role in central hypoventilation syndromes. The sudden infant death syndrome (SIDS) involves a failure of respiration and affects infants in their early postnatal months. Unstable respiratory activity during sleep, prolonged sleep apnea, and oropharyngeal/laryngeal dysfunction induced by liquid stimulation of the upper airways have been postulated to be implicated in its genesis. It has also been found that in comparison with normal infants, infants suffering from the "aborted syndrome" (near-miss infants) are hypoventilated during quiet sleep, and have an impaired ventilatory response to carbon dioxide breathing.
\end{abstract}

Keywords: Peripheral chemoreceptors, aorticopulmonary paraganglia, carotid body, SIDS, stillbirth.

\section{INTRODUCTION}

The efficient uptake and delivery of oxygen and removal of carbon dioxide are critical to healthy survival, and a host of mechanisms are therefore deputed to achieving this goal. After birth, these mechanisms are directed at both the cardiovascular and pulmonary systems, whereas prior to birth the lungs play no role in gas exchange. Thus, it is likely that any mechanism that regulates the oxygen and acid-base status will function quite differently in the fetus, and that birth is associated with a sudden and dramatic alteration in its function. The mechanism involved consists of chemoreception and transduction, a process whereby alterations in oxygen, carbon dioxide and acid-base status are sensed by receptors, which then invoke a variety of responses to mitigate these alterations. Although innumerable studies have been performed since De Castro [1,2] and Heymans and coworkers [3] first began the study of peripheral chemoreceptor function, and Barcroft [4] then extended these studies to the fetus, much of the issue is still speculative and should be considered as an aid to focusing future studies rather than as a repository of fact. In his presentation to the International Society for Arterial Chemoreception in 1991, Eyzaguirre [5] summed up the current understanding of chemoreception most aptly: "this field will remain puzzling until more information on different variables is available."

This review will focus firstly on the basic mechanisms of the peripheral chemoreceptors elicited by studies on mature

\footnotetext{
*Address correspondence to this author at the Chairman, Institute of Pathology, University of Milan, Via della Commenda, 19, 20122 Milan, Italy; Tel: +39-02-5419521; Fax: +39-02-5419538; E-mail: luigi.matturri@unimi.it
}

chemoreceptors. It then will address the alterations observed in these structures related to sudden infant death.

\section{CHEMORECEPTION: BASIC MECHANISMS}

\section{Anatomy}

The components of chemoreception include peripheral and central neuroreceptors, neural afferents, and neurohumoral effectors that alter both pulmonary performance (rate and depth of breathing) and cardiovascular performance (ventricular performance, regional blood flows and perfusion pressures). The peripheral chemoreceptors are composed of glomera, or tissues with extensive and convoluted capillary networks. Although there is glomus tissue in a variety of sites throughout the body, the most important are the carotid body, located at the junction of the external and internal carotid arteries, and the aortic bodies (also known as aorticopulmonary paraganglia), a more diffuse group of small glomera primarily situated in the aortic arch. In addition to the network of capillaries, the glomus consists of three cell types: the type I, or glomus cell, thought to be the true receptor, is predominant; the type II, or sustentacular cell, functions as the support cell and features extensive glial-like projections; and few ganglion cells are present, which may be either sympathetic or parasympathetic [6]. The carotid body is innervated by the carotid (sinus) nerve, which is a branch of the glossopharyngeal nerve, while the aortic bodies are innervated by the aortic nerve, a branch of the vagus. These nerves carry autonomic traffic for both the chemoreceptors and the adjacent baroreceptors. In the glomera, they branch extensively, innervating 10-20 glomus cells, which form the sensory unit [7]. Each fiber may distribute both afferent or post-synaptic nerve endings, the junctions of which are characterized by the secretory 
vesicles residing in the glomus cells and efferent or presynaptic nerve endings, where the secretory vesicles are directly resident. Efferent fibers may modulate receptor function by secreting neuromodulators which alter glomus cell function, or by altering blood flow to the sensory unit, which in turn alters the stimulus to the receptor discharge [7].

The central chemoreceptor components are less well described. Over the past decade, an increasing number of central nuclei, in addition to the ventrolateral medulla, have been proposed to contain putative central chemoreceptors, including the solitary complex, the medullary raphe, the nucleus ambiguus, the locus coeruleus, and the hypothalamus [8]. Recent pathological investigations of sudden fetal deaths after the $25^{\text {th }}$ week of gestation (stillbirths) have demonstrated frequent hypoplasia of the arcuate nucleus, an important chemoreceptorial respiratory center of the medullary ventral surface $[9,10]$. This had previously been described in sudden infant deaths [11]. Further research is necessary and ongoing to identify any links between abnormalities of the central and peripheral chemoreceptor apparatus with central hypoventilation syndromes, such as sleep apnea and sudden infant death syndrome.

\section{Integrated Physiology}

The integrated response of the peripheral chemoreceptors lies in the transduction of alterations in the chemical milieu, regulating the tonicity, or the temperature of their environment to alterations in cardiovascular and respiratory performance [7]. Although the carotid chemoreceptors respond to changes in both oxygen and acid-base status, the aortic chemoreceptors are much more sensitive to oxygen, and as such have been considered to have the physiological function of oxygen sensors [12,13]. The sensory discharge from both chemoreceptors reaches the respiratory and cardiovascular centers in the brainstem, and invokes increases in ventilation, heart rate and cardiac output, as well as peripheral vasoconstriction through autonomic pathways and catecholamine release from the adrenal medulla. Superimposed on the cardiorespiratory effects of the peripheral chemoreceptors, there are the independent effects of the baroreceptors and central chemoreceptors [6]. The central chemoreceptors respond primarily to changes in $\mathrm{pCO}_{2}$ and alter ventilation by direct effects on the respiratory center, while the baroreceptors respond to changes in local arterial pressure and alter regional blood flows by sympathetic stimulation and secretions of various hormones, such as ACTH, vasopressin and renin; these hormonal responses are not apparently directly induced by chemoreceptor activity [14].

\section{Biological Mechanisms}

The subcellular mechanisms of various components of chemoreception and transduction have been extensively studied over the past several years. The components can be classified as follows: the sensory components, in which the stimulus is perceived by the sensing organ as having changed; the intracellular components, in which a series of intracellular events then triggers the release of neurotransmitters; the afferent neural components, in which the neurotransmitters affect the sensory discharge of the local fibers; the efferent neural components, which respond to changes in sensory input with alterations in their activity; and the cardiorespiratory components, which respond to changes in the activity of the effector nerves with changes in cardiac, vascular and respiratory performance [15].

The sensory components of the peripheral and central chemoreceptors have been extensively studied, but although a large body of information is now available, many data and hypotheses are contradictory. It is not even entirely clear which cells in the glomera comprise the sensory unit, although it is now generally accepted that they are the type I cells. The mechanisms underlying stimulation of the sensory unit remain unclear. The discrepancy in the sensitivity of the carotid and the aortic bodies to oxygen is explained in this model: the carotid body is postulated to have very high blood flow and oxygen metabolism, and thus to be insensitive to modest changes in blood pressure or oxygen content, whereas the aortic body, with its lower flow, may be very sensitive to even modest changes in these variables $[16,17]$. This hypothesis is taken further to assume different roles for these two main peripheral chemoreceptors: the sensitivity of the aortic body to modest circulatory changes makes it well suited to functioning as the monitor of the combined state of respiratory oxygen uptake and cardiovascular oxygen delivery [18], while the lack of sensitivity to changes in $\mathrm{pCO}_{2}$ places it as the sensor of respiratory oxygen uptake [17]. It is clear that a variety of chemicals are released by chemoreceptor stimulation, including catecholamines, acetylcholine, and neuropeptides, but the importance of each in response to a given stimulus or even a given species is uncertain [5]. Catecholamines, particularly dopamine, may also play an important role as neurotransmitters [19]. Granules containing catecholamines are present in high concentrations in the carotid body, particularly during chronic hypoxia [20], and chemodectomas occur far more commonly at high altitude [21]. Whatever may be the precise mechanisms underlying neurotransmitter release and its modulation, the release invokes a nerve-cell discharge which ascends to the nucleus tractus solitarius and, via a change in the frequency or profile of the discharge, elicits cardiopulmonary reflexes.

\section{Chemoreception in the Fetus}

Perhaps more than any other vital activity, the maintenance of adequate oxygen uptake and delivery differs profoundly in fetal life in comparison with the means adopted after birth. Oxygen uptake is dependent upon adequate blood flow and gas exchange across the placenta, arising from the descending aorta. Oxygen is delivered by both ventricles: the inflow of oxygenated blood from the umbilical circulation is partially delivered to the left ventricle across an intracardiac shunt, the foramen ovale; and the outflow of oxygenated blood from the right ventricle is delivered to the systemic vascular bed across an extracardiac shunt, the ductus arteriosus. It is not therefore surprising that chemoreception must evoke very different cardiopulmonary responses in the fetus. When the study of fetal chemoreception began about fifty years ago, it was generally believed that the absence of effective ventilation in utero implied that chemoreception was of limited importance to the fetus. It was long believed that dramatic maturation 
occurred in the perinatal period, allowing the newborn to respond to hypoxia and hypercapnia by increasing ventilatory effort. Although the sensory neurons which form the afferent limb of the chemoreflex have been demonstrated to be biochemically immature [22], it is becoming apparent, however, that the chemoreceptor response is quite mature in the late-term fetus [15].

After birth, the inhibition of continuous breathing is immediately abolished and peripheral chemoreceptors assume their major role in the regulation of breathing. It would be logical to speculate that removal of the placental factor is responsible [23]. Another hypothesis is that the activity of the peripheral chemoreceptor, particularly the carotid body, increases dramatically as blood flow decreases [24], thereby overwhelming the inhibitory effects of the lateral pons. However, a recent study has demonstrated that in about a third of stillbirths there is congenital hypodevelopment of both the lung and the arcuate nucleus[25,26]. It was postulated that in these cases the arcuate nucleus hypoplasia could exert a negative effect on respiratory movements in utero and therefore on lung development. These alterations support the hypothesis that functional or structural abnormalities of components of the vegetative nervous system that modulate fetal breathing lead to disturbances in the development of the respiratory apparatus, in particular pulmonary hypoplasia in stillbirth.

Over the years, much has been written about the relative importance of the carotid and aortic bodies in the peripheral chemoreceptor response of the fetus, and it is therefore worthwhile to consider the current state-of-the-art on the issue. Because the postnatal activity of the carotid body is primarily directed toward maintaining adequate ventilation via $\mathrm{O}_{2}$ and $\mathrm{CO}_{2}$ sensing and the aortic bodies are primarily directed toward maintaining adequate oxygen delivery via $\mathrm{O}_{2}$ sensing, it was originally hypothesized that the aortic chemoreceptors were much more important than the carotid chemoreceptors in the fetus. Initial studies seemed to confirm this hypothesis, suggesting that the aortic chemoreceptors were largely responsible for the cardiovascular response to hypoxia in late-gestation fetal sheep [27]. The difference lies not in the activity of the carotid chemoreceptor but in the ventilatory response to that activity, which is suppressed by central inhibition of the hypoxic ventilatory response prior to birth.

Several lines of evidence have suggested that abnormalities in chemoreception may play a role in central hypoventilation syndromes [9-11,28]. The sudden infant death syndrome involves a failure of respiration and affects infants in their early postnatal months. Unstable respiratory activity during sleep, prolonged sleep apnea, and oropharyngeal/laryngeal dysfunction induced by liquid stimulation of the upper airways have been postulated to be implicated in its genesis [29-31]. It has also been found that in comparison with normal infants, infants suffering from the "aborted syndrome" (near-miss infants) are hypoventilated during quiet sleep, and have an impaired ventilatory response to carbon dioxide breathing during quiet sleep [32]. The underlying mechanisms are unknown but there is presumably some malfunction in the respiratory control if breathing ceases during sleep, and if arousal and hyperventilation do not occur despite the development of asphyxia in these infants.

\section{THE SUDDEN INFANT DEATH SYNDROME (SIDS)}

\section{Clinical and Epidemiological Features}

Crib death or the Sudden Infant Death Syndrome (SIDS) is the major cause of death in infants within the first year of age [33]. According to the definition proposed by Beckwick [34], SIDS is the sudden death of an infant or young child which is unexpected in view of its medical history, and in which a thorough postmortem examination fails to demonstrate an adequate cause of death. This definition implies that most diagnoses of SIDS are made postmortem, and by exclusion. However, in the clinical history of infants dying of SIDS it is common to find episodes of cyanosis and apnea while the babies were quietly sleeping, and there are vivid descriptions of "near misses" in which babies have been resuscitated from episodes of prolonged apnea.

The cause of death in SIDS may be the result of multiple determining factors, and of an interaction between physiological and environmental elements. SIDS is rare in babies less than 1 month and over 6 months of age, having a peak incidence at 2-4 months of age. This epidemiological fact might suggest that there is a particularly vulnerable stage in the development of respiratory control between these ages [35]. Infants with low weight at birth, or born prematurely, are at increased risk of SIDS, as are infants of multiple births and newborns of human newborns in comparison with other animal orders [36]. The incidence of SIDS varies with the season, with more deaths occurring during the winter months, suggesting that there may be some infectious component in triggering SIDS. Maternal risk factors include: young maternal age, poor prenatal care, bleeding during pregnancy, and smoking. Furthermore, there is evidence that household exposure to tobacco smoke has an independent additive effect [37,38]. Parental drug misuse (apart from smoking) has an additional small but significant effect [39]. These and other environmental factors, such as infections for example, may act in combination with a vulnerable stage of physiological development, to precipitate death [40].

SIDS is closely associated with sleep. The relationship between SIDS and the sleeping position has been examined in several epidemiological studies. Most, but not all, of these studies found that the prone position during sleep was more common in babies dying of SIDS than in control babies. The mechanism underlying the apparently significant association between the prone position for sleep and the risk for SIDS is unknown, but impaired ventilatory control and arousal responsiveness have been suggested [41].

\section{Peripheral Chemoreceptors and SIDS}

Several hypotheses have proposed the implication of a defective function of the peripheral chemoreceptors in the etiology of SIDS ${ }^{28,42}$. Over the last 30 years, the morphology of the carotid body has been addressed in several studies on SIDS. In general, however, the findings have been conflicting. Naeye et al. found abnormally increased or abnormally decreased amounts of glomic tissue to be present, the former being more prominent in victims with more severe antecedent chronic hypoxia [42]. Lack et al. 
observed that the carotid body combined weights were slightly heavier in SIDS cases, but computerized planimetry of the total surface area and the area occupied by "functional parenchyma" revealed no significant differences between SIDS victims and controls ${ }^{41}$. Furthermore, the chief cells showed a similarly intense degree of cytoplasmic argyrophilia in both groups [42] and ultrastructural studies yielded contradictory results [43-45]. Although these data did not support a significant role for the carotid body in the pathogenesis of SIDS, further investigation of this issue is warranted.

The specific function of the vagal body paraganglia, located at various points along the peripheral distribution of the vagus nerve, is still unclear [6]. These consist of small groups of glomic tissue, typically situated inside the perineurium, just beneath the nerve sheath or between nerve fibers, which cannot be microscopically or cytochemically distinguished from the other peripheral chemoreceptors [46]. Hyperplasia of the vagal body paraganglia has been noted in patients with chronic hypoxemia, supporting a chemoreceptor function for this neuroendocrine tissue [47]. In SIDS, no significant microanatomic differences have been reported compared with controls, but an underlying functional abnormality with autonomic dysfunction cannot be excluded [46].

Located within the epithelial lining of the pulmonary airways, the neuroepithelial bodies are considered intrapulmonary chemoreceptors. Compensatory hyperplasia of the neuroepithelial bodies has been described in the lungs of SIDS victims, characterized by an increase in the frequency, size and mean concentration of bombesin-like peptides of the neuroepithelial cells, when compared to the values in age-matched controls [48,49]. Furthermore, the frequency and size of pulmonary neuroepithelial bodies immunostained for bombesin was increased twofold in two cases of the congenital central hypoventilation syndrome, a rare disorder of unknown etiology characterized by failure of respiratory control [50]. Since neuroepithelial bodies are thought to function as hypoxia-sensitive airway chemoreceptors, it has been speculated that chronic hypoxia and/or brainstem dysfunction may be responsible for this alteration, contributing to the pathophysiology of SIDS and related conditions such as the congenital central hypoventilation syndrome [48-50].

Enlargement of the aorticopulmonary paraganglia has been described in some infants who died of SIDS [28,51,52]. This alteration, present in $24 \%$ of SIDS cases, can easily be inferred from sections under a low power microscope lens, as shown in (Fig. 1), nevertheless, unequivocal proof of glomic cell proliferation can only be gained by morphometrical measurement. The normal appearance of a few, small, discrete lobules separate from the fibroadipose tissue is lost and individual lobules show great enlargement with irregular and elongated profiles. The enlargement of the aorticopulmonary paraganglia is due in a small part to an increase in the number of lobules but more importantly, to an increase in size of the lobules. The cell clusters and cell diameters have not been found to be significantly different from those in age-matched controls, thus enlargement of the aorticopulmonary paraganglia involves an increase in the number rather than the size of cells, favoring hyperplasia rather than hypertrophy [28].

Previous reports had described a more extensive and prominent distribution of paraganglion cells in the human fetus and in newborn infants than in adults [6,53]. Our studies have confirmed this finding [28]. The aorticopulmonary paraganglia were more developed in controls at birth and decreased dramatically during the first year of life. The mechanisms underlying this morphologic variation are not yet known [54]. This normal "shaping or molding" with age was not observed in the SIDS group. The aorticopulmonary paraganglia volumes in these cases appeared to be already increased in the younger group and remained practically unaltered at all age intervals, suggesting that the stimulus to proliferate (hypoxia, trauma or irritation) could be produced or initiated during fetal development or in the early postnatal period. This stimulus could produce structural and/or functional alterations in the chemoreceptor cascade in the late-term fetus or newborn infant, preventing them from responding promptly to chemical stimuli [28].

\section{Final Comments}

The core of the SIDS physiopathology is attributed to impaired developmental processes in chemoreceptors, including their neurotransmitter systems, in both the peripheral and the central nervous system. Some environmental factors have a well-established role in the genesis of such alterations. At the functional level, SIDS implies a defective resetting (slow or incomplete) of the sensitivity to hypoxia and hypercapnia and/or a defective processing of signals in the cardiorespiratory centers of the brainstem. A normal infant's nervous system receives prompt information on a progressive hypercapnia and hypoxia event, and triggers a new respiration or arousal; in infants with peripheral or central chemoreception defects these protective reflexes are not initiated at normal levels of hypoxia and hypercapnia, and prolonged apnea can develop until blood gas alterations reach the higher level required to initiate the new respiration or arousal. These prolonged apnea may lead to fatal apnea and sudden death.

On the other hand, some victims of SIDS present higher iron concentrations in the liver than those in normal newborns [55]. It is not presently known whether the increase in blood iron and ferritin concentrations is a cause or an effect (or is an unrelated finding) of abnormal oxygen management, including altered sensitivity to low oxygen.

These findings suggest the possibility that in SIDS cases, regardless of the quality of the central nervous system, the final effectors of respiratory output might be too inefficient to respond. However, whether these alterations are unique or primary in triggering SIDS is not known.

In conclusion, it is important to submit every case of sudden unexpected infant death to complete and accurate post mortem investigation, including close study of the peripheral chemoreceptors. In fact, the current definition of SIDS as "the sudden death of an infant under one year of age which remains unexplained after a thorough case investigation, including performance of a complete autopsy, examination of the death scene, and review of the clinical history" needs to be amplified, to include "complete autopsy examination 

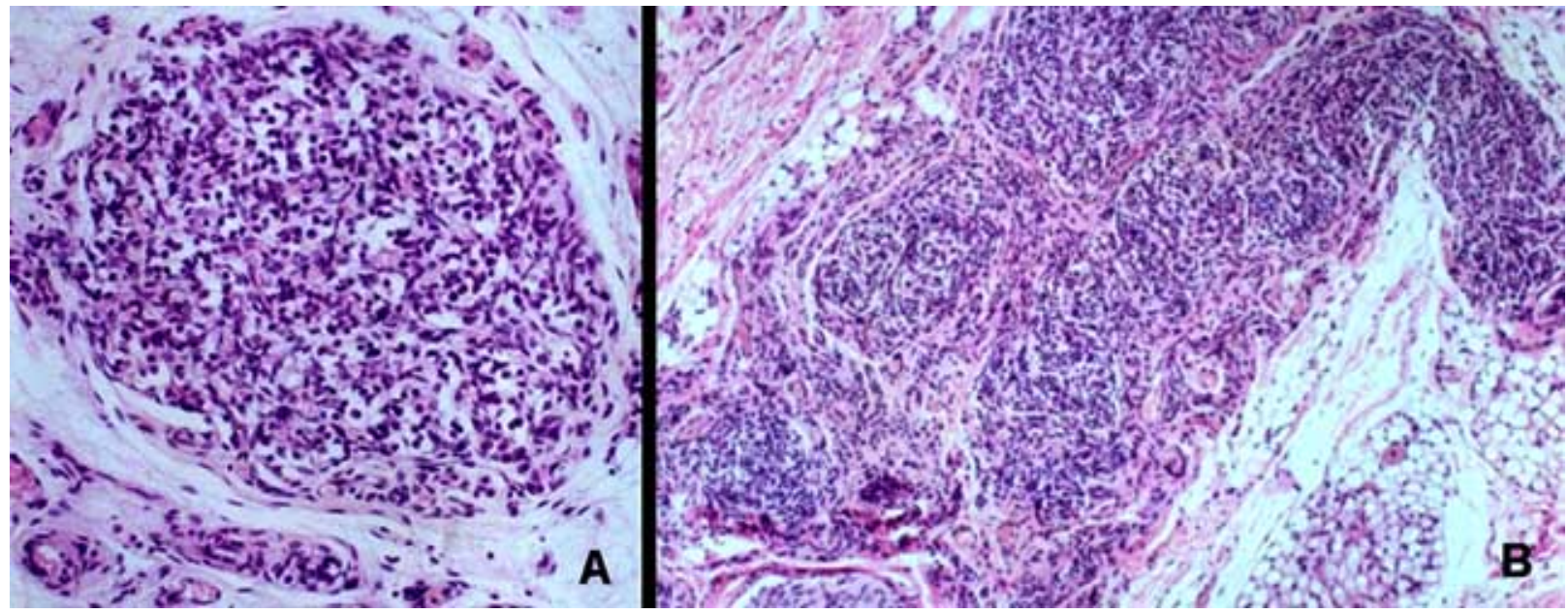

A.) Small lobule of aorticopulmonary paraganglia from a 180-day-old female infant who died during an acute rejection of liver transplant, showing clusters of chief cells, with slightly predominance of light variant (arrows) which had indistinct cytoplasmic borders in most areas. The light cells were intercaled by dark cells (head arrows) in clusters surrounded by sustentacular cells (straight arrows) (hematoxilin-eosin, original magnification 400x).

B.) Great enlargement of the aorticopulmonary paraganglia (arrows) from a 66-day-old female infant victim of sudden infant death syndrome. One can appreciate sprinkling of small, dot-like cells at this magnification which represents chief cells. The aorticopulmonary paraganglion volume was $0,16 \mathrm{~mm}^{3}$. The glomic tissue shows prominent dark cells (head arrows) exhibiting a compact basophilic cytoplasm, often in strap-like cytoplasmatic extensions with eccentric and hyperchromatic nuclei. The cores of dark cells became spaced, leaving fewer chief cells per unit area when compared with the controls in spite of hyperplasia. The clusters were surrounded by sustentacular cells (straight arrows) (hematoxilin-eosin, original magnification 250x).

with an in-depth histopathological analysis of the autonomic nervous system and of the cardiac conduction system performed by an experienced, reliable pathologist" [56].

\section{ACKNOWLEDGEMENTS}

The authors thank Marina Crippa and Graziella Alfonsi for their excellent technical assistance. Dr. Simone G. Ramos is an investigator of National Council of Scientific and Technological Development (CNPq), Brazil.

This study was supported by the Ministry of Foreign Affairs (joint project of particular relevance $n^{\circ}$ 269/P/0085087 "Anatomopathologic and genetic study of the unexplained perinatal death and SIDS") and by the "Lino Rossi" Research Center for the study and prevention of unexpected perinatal death and sudden infant death syndrome (SIDS) funding (Rectorial Decree n. 225678 of 23/04/04).

\section{REFERENCES}

[1] De Castro F. Sur la structure et l'innervation de la glande intercarotidienne (glomus caroticum) de l'homme et des mammifères et sur un nouveau système d'innervation autonome du nerf glossopharyngien. Études anatomiques et physiologiques. Trab Lab Invest Biol Univ Madrid 1926; 24: 365-432.

[2] De Castro F. Sur la structure et l'innervation du sinus carotidien de l'homme et des mammifères. Nouveaux faits sur l'innervation et la fonction du glomus caroticum. Études anatomiques et physiológiques. Trab Lab Invest Biol Univ Madrid 1928; 25: 33180.

[3] Heymans C, Bouchaert JJ, Dautebrande L. Sinus carotidien et reflexes respiratoires. II. Influences respiratoires réflexes de l'acidose, de l'alcalose, de l'anhydride carbonique, de l'ion hydrogène et l'anoxémie. Sinus carotidien et échanges respiratoires dans les poumons et au dela des poumons. Arch Int Pharmacodyn Thér 1930; 39: 400-50.
[4] Barcroft J. Researches on Pre-Natal Life. Vol. 1. Oxford, Blackwell Scientific Publications Ltd. 1946.

[5] Eyzaguirre C. Those strange glomus cells. In: Neurobiology and Cell Physiology of Chemoreception. Ed.: P. Data, H. Acker and S. Lahiri. New York, Plenum Press. 1993; 123-9.

[6] Glenner GG, Grimley PM. In: Tumors of the extra-adrenal paraganglion system (including chemoreceptors). 2nd ed. Washington, Armed Forces Institute of Pathology. 1974.

[7] Eyzaguirre C, Fitzgeralg RS, Lahiri S, Zapata P. Arterial chemoreceptor. In: Sheperd T. and Abboud. FM. Ed.: Handbook of Physiology: The Cardiovascular System. Vol. III, part 2. Bethesda, American Physiologic Society. 1983; 557-621.

[8] Ballantyne DJ, Dean JB, Putnam RW. Central chemosensitivity. Resp Physiol 2001; 129: 1-3.

[9] Matturri L, Minoli I, Lavezzi AM, Capellini A, Ramos S, Rossi L. Hypoplasia of medullary arcuate nucleus in unexpected late fetal death (stillborn infants): a pathologic study. Pediatrics 2002; 109: E43.

[10] Matturri L, Ottaviani G, Alfonsi G, Crippa M, Rossi L, Lavezzi AM. Study of the brainstem, particularly the arcuate nucleus, in sudden infant death syndrome (SIDS) and sudden intrauterine unexplained death (SIUD). Am J Forensic Med Pathol 2004; 25: 44-8.

[11] Matturri L, Biondo B, Suarez-Mier MP, Rossi L. Brainstem lesions in the sudden infant death syndrome: variability in the hypoplasia of the arcuate nucleus. Acta Neuropathol 2002; 104: 12-20.

[12] Anand A, Painal AS. Oxygen sensing by arterial chemoreceptors. In: Response and Adaptation to Hypoxia: Organ to Organelle. Ed.: S. Lahiri, N.S. Cherniak and R.S. Fitzgerald. New York, Oxford University Press. 1991; 81-94.

[13] Mokashi A, Lahiri S. Aortic and carotid body chemoreception in prolonged hyperoxia in the cat. Resp Physiol 1991; 86: 211-4.

[14] Chen HG, Wood CE. Reflex control of fetal arterial pressure and hormonal responses to slow hemorrhage. Am J Physiol 1992; 262: H225-33.

[15] Teitel DF. Fetal chemoreception: a developing story. Reprod Fertil Dev 1996; 8: 471-82.

[16] Lahiri S. Role of arterial $\mathrm{O}_{2}$ flow in peripheral chemoreceptor excitation. Fed Proc 1980; 39: 2648-52.

[17] Lahiri S. Oxygen biology of peripheral chemoreceptors. In: Response and Adaptation to Hypoxia: Organ to Organelle. Ed.: S. 
Lahiri, N.S. Cherniak and R.S. Fitzgerald. New York, Oxford University Press. 1991; 95-121.

[18] Lahiri S, Nishino T, Mokashi A, Mulligan E. Relative responses of aortic body and carotid body chemoreceptors to hypotension. J Appl Physiol 1980; 48: 781-8.

[19] Fidone SJ, Gonzalez C, Yoshizaki K. Putative neurotransmitters in the carotid body: the case for dopamine. Fed Proc 1980; 39: 263640.

[20] Arias-Stella J, Valcarcel J. Chief cell hyperplasia in the human carotid body at high altitudes: physiologic and pathologic significance. Hum Pathol 1976; 7: 361-73.

[21] Arias-Stella J, Bustos F. Chronic hypoxia and chemodectomas in bovines at high altitudes. Arch Pathol Lab Med 1976; 100: 636-9.

[22] Katz DM. Molecular mechanisms of carotid body afferent neuron development. In: Lahiri S. Cherniak NS. and Fitzgerald RS. Eds.: Response and Adaptation to Hypoxia: Organ to Organelle. New York, Oxford University Press. 1991; 133-42.

[23] Donnelly DF, Haddad GG. Prolonged apnea and impaired survival in piglets after sinus and aortic nerve section. J Appl Physiol 1990; 68: $1048-52$.

[24] Jansen AH. Peripheral chemoreceptor function in the fetus. Sem Perinatol 1977; 1: 327-37.

[25] Matturri L, Lavezzi AM, Minoli I, et al. Association between pulmonary hypoplasia and hypoplasia of arcuate nucleus in stillbirth. J Perinatol 2003; 23: 328-32.

[26] Lavezzi AM, Ottaviani G, Rossi L, Matturri L. Hypoplasia of the Parabrachial/Kölliker-Fuse complex in perinatal death. Biol Neonate 2004; 86: 92-7.

[27] Dawes GS, Duncan SL, Lewis BV, Merlet CL, Owen-Thomas JB, Reeves JT. Hypoxaemia and aortic chemoreceptor function in foetal lambs. J Physiol 1969; 201: 105-16.

[28] Ramos SG, Matturri L, Biondo B, Ottaviani G, Rossi L. Hyperplasia of the aorticopulmonary paraganglia: a new insight into the pathogenesis of sudden infant death syndrome? Cardiologia 1998; 43: 953-8.

[29] Cherniak NS. Sleep apnea and its causes. J Clin Invest 1984; 73: 1501-06.

[30] Naeye RL. Hypoxemia and sudden infant death syndrome. Science 1974; 186: 837-8.

[31] Steinschneider A, Weinstein SL, Diamond E. The sudden infant death syndrome and apnea obstruction during neonatal sleep and feeding. Pediatrics 1982; 70: 858-63.

[32] Shannon DC, Kelly DH, O'Connel K. Abnormal regulation of ventilation in infant at risk for sudden infant death syndrome. $\mathrm{N}$ Engl J Med 1977; 297: 747-50.

[33] Finlay FO, Rudd PT. Current concepts of the aetiology of SIDS. Br J Hosp Med 1993; 49: 727-32.

[34] Beckwith JB. Discussion of terminology and definition of Sudden Infant Death Syndrome. In: Proc Second Int Conf Causes Sudden Death Infants. Eds.: AB Bergman, JB Beckwith, CG Ray. Seattle, University of Washington 1970; 14-22.

[35] Takashima S, Mito T, Yamanouchi H. Development brain-stem pathology in sudden infant death syndrome. Acta Paediatr Jap 1994; 36: 317-20.

[36] Guntheroth WG, Spiers PS, Naeye RL. Redefinition of the sudden infant death syndrome: the disadvantages. Pediatr Pathol 1994; 14: 127-32.

[37] Naeye R, Ladis B, Drage J. Sudden infant death syndrome: a prospective study. Am J Dis Child 1976; 130: 1207-10.
[38] Taylor JA, Sanderson M. A reexamination of the risk factors for the sudden infant death syndrome. J Pediatr 1995; 126: 887-91.

[39] Blair PS, Fleming PJ, Bensley D, et al. Smoking and the sudden infant death syndrome: results from 1993-5 case-control study for confidential inquiry into stillbirths and deaths in infancy. Br Med J 1996; 313: 195-8.

[40] Golding J, Limerick S, Macfarlane A. Summary of epidemiological findings. In: Sudden Infant death: Patterns, Puzzles and Problems. Eds.: J Golding, S Limerick, A Macfarlane. Shepton Mallet, England, Open Book. 1985; 38-40.

[41] Hunt CE, Shannon DC. Sudden infant death syndrome and sleeping position. Pediatrics 1992; 90: 115-8.

[42] Naeye RL, Fisher R, Ryser M, Whalen P. Carotid body in the sudden infant death syndrome. Science 1976; 191: 567-9.

[43] Lack EE, Perez-Atayde AR, Young JB. Carotid body in the sudden infant death syndrome: A combined light microscope, ultrastructural, and biochemical study. Pediatr Pathol 1986; 6: 33550.

[44] Cole S, Lindenberg LB, Galeoto FM Jr, et al. Ultrastructural abnormalities of the carotid body in sudden infant death syndrome. Pediatrics 1979; 63: 13-7.

[45] Perrin DG, Cutz E, Becker LE, Bryan AC. Ultrastructure of carotid bodies in sudden infant death syndrome. Pediatrics 1984; 73: 64651.

[46] Lack EE. Microanatomy of vagal body paraganglia in infancy including victims of sudden infant death syndrome. Pediatr Pathol 1989; 9: 373-86.

[47] Lack EE. Hyperplasia of vagal and carotid body paraganglia in patients with chronic hypoxemia. Am J Pathol 1978; 91: 497-516.

[48] Gillan JE, Curran C, O'Reilly E, Cahalane SF, Unwin AR. Abnormal patterns of pulmonary neuroendocrine cells in victims of sudden infant death syndrome. Pediatrics 1989; 84: 828-34.

[49] Perrin DG, MacDonald TJ, Cutz E. Hyperplasia of bombesinimmunoreative pulmonary neuroendocrine cells and neuroepithelial bodies in sudden infant death syndrome. Pediatr Pathol 1991; 11: 431-47.

[50] Cutz E, Ma TKF, Perrin DG, Moore AM, Becker LE. Peripheral chemoreceptors in congenital central hypoventilation syndrome. Am J Respir Crit Care Med 1997; 155: 358-63.

[51] Rossi L, Matturri L. Anatomohistological features of the heart's conduction system and innervation in SIDS. In: Rognum TO, ed. Sudden infant death syndrome: New trends in the nineties. Oslo, Scandinavian University Press. 1995; 207-12.

[52] Matturri L, Migliarini AM, Rossi L. Changes in the cardiovascular chemo-baroreceptors as a possible basis for sudden infant death syndrome (SIDS). Pathologica 1992; 84: 467-71.

[53] Gilson TP, Balko MG, Blisard KS, Taylor KL. Morphologic variations of the external arcuate nucleus in infants dying of SIDS: A preliminary report. J Forensic Sc 1994; 39: 1076-83.

[54] Hervonen A, Vaalasti A, Partanen M, Kanerva L, Vaalasti T. The paraganglia, a persisting endocrine system in man. Am J Anat 1976; 146: $207-10$.

[55] Raha-Clowdhury R, Moore CA, Bradley D, Henley R, Worwood $\mathrm{M}$. Blood ferritin concentrations in newborns infants and the sudden infant death syndrome. J Clin Pathol 1996; 49: 168-70.

[56] Matturri L, Lavezzi AM, Rossi L. Proposal to modify the definition of SIDS, with regard to the post-mortem exam. In: Florence, Italy, Proceed. $7^{\text {th }}$ International Conference on SIDS. 2002; 103. 